\title{
Gravitational Waves and Neutron Stars
}

\author{
Bernard F. Schutz \\ Albert Einstein Institute, Potsdam, Germany \\ Cardiff University, Wales, UK \\ schutz@aei-potsdam.mpg.de
}

\begin{abstract}
The first generation of laser-interferomteric gravitational wave observatories will make intensive searches for gravitational radiation from spinning neutron stars. Sensitivity to a number of possible sources, including the Crab pulsar, will be better than any existing observational limits, and will improve dramatically over the next decade. This paper reviews these developments and expectations, and discusses ways in which pulsar radio astronomers and gravitational wave astronomers can benefit from one another's work.
\end{abstract}

\section{Introduction}

After 4 decades of development, gravitational wave detectors will soon reach a sensitivity where the first detections could occur, and there is a good chance that neutron stars will be well represented among the first detected sources. By 2001 the American LIGO detectors (two 4-km interferometers, see Raab 1995) and the German-British GEO600 detector (one 600-m instrument, see Danzmann 1995) should be sensitive to bursts of amplitude around $10^{-21}$ and continuous waves of amplitude $10^{-26}$. Within a further year, the Italian-French VIRGO detector (one 3-km detector, see Giazotto 1995) may come on-line with an even better sensitvity. They build on the deep foundation that pulsar astronomy has given to theoretical physics: observations of the Hulse-Taylor pulsar PSR $1913+16$ assure us that gravitational wave theory is sound (Taylor 1994).

However, while early detections at this level are possible, they are by no means assured. For example, if the Crab pulsar emits gravitational waves that carry away only one part in $10^{-4}$ of the total energy it is losing as it spins down, then the first detectors should see it; but there is no compelling reason to expect this.

Data analysis for gravitational waves from pulsars has much in common with radio pulsar search analysis. The frequencies of the signals are similar (neutron stars radiate gravitational waves at frequencies between one and two times their spin frequencies), and the parameter space that must be searched is large. Positions will be determined by fitting phase arrival times over periods as long as a year, just as for radio observations, so the angular accuracy of positions will be similar. But, unlike radio observations, gravitational wave detectors need to observe continuously for months; the signal will not have enough power to allow us to detect a source with only a few hour-long observations. And detectors 
are almost omni-directional; "pointing" is done by removing phase modulation in the data analysis.

The earliest detections will probably have small signal-to-noise ratios, which demands that we use the best signal analysis methods. The optimum linear method is matched filtering (Helström 1968) in which the computer looks for a correlation in the noisy data with a template, which is an expected waveform. For some sources, such as coalescing compact-object binary systems (Blanchet et al. 1995) and spinning neutron stars (Brady et al. 1998), we believe we have good theoretical templates. However, in all cases the waveform templates depend on parameters that may not be known ahead of time, such as the location on the sky of the neutron star and the first few time-derivatives of its frequency. Using these templates becomes a compute-intensive job, and the algorithms must be implemented as efficiently as possible.

\section{Gravitational Wave Detectors}

The detectors now under construction are just first-stage instruments, which have been designed simply to cross the critical threshold of $10^{-21}$ where detections are possible but not assured. It has always been part of the planning for these detectors that sensitivity upgrades would follow swiftly. In part by using GEO technology, LIGO has designs for

a factor of 10 improvement in amplitude sensitivity in 5 years. VIRGO will also implement GEO technology to upgrade its initial design sensitivity. On the same timescale, a $3-\mathrm{km}$ detector may also be built in Japan, a successor to the prototype TAMA300 detector in Tokyo (Tsubono 1995), which at present is the most sensitive interferometer that has so far been operated. By 2010 a further improvement of 10 in amplitude sensitivity is possible. Gravitational wave astronomy will reach maturity at that stage.

\section{Gravitational Waves From Neutron Stars - General Considera- tions}

The radiation from a spinning neutron star will emerge at a frequency between 1 and 2 times spin frequency, depending on the radiation mechanism (next section). Detectors, observing coherently, will measure polarization, phase, and amplitude. The waves do not scatter and are not absorbed between the source and the detector, but they can be gravitationally lensed.

The amplitude is impossible to predict for most sources. For normal pulsars, for example, we only have upper limits set by the observed slowdown, as discussed below: the energy carried by the radiation should not be larger than the kinetic energy lost as the pulsar spins down. In fact the emission may be much smaller than this limit.

In Figure 1 we show the 1-year sensitivity of the GEO600 detector against the spindown upper limits for a number of known pulsars, adjusted for the average antenna pattern of GEO600 detector for each. The Crab stands well above the noise in this figure, and PSR J0540-6919 is also in the detection region. For each pulsar the figure also shows the amplitude it would radiate if its ellipticity were as small as $10^{-5}$, which is regarded as a plausible level that 
the crust of a neutron star might sustain for long periods of time. Note that the millisecond pulsars have spindown limits that need smaller ellipticities than this.

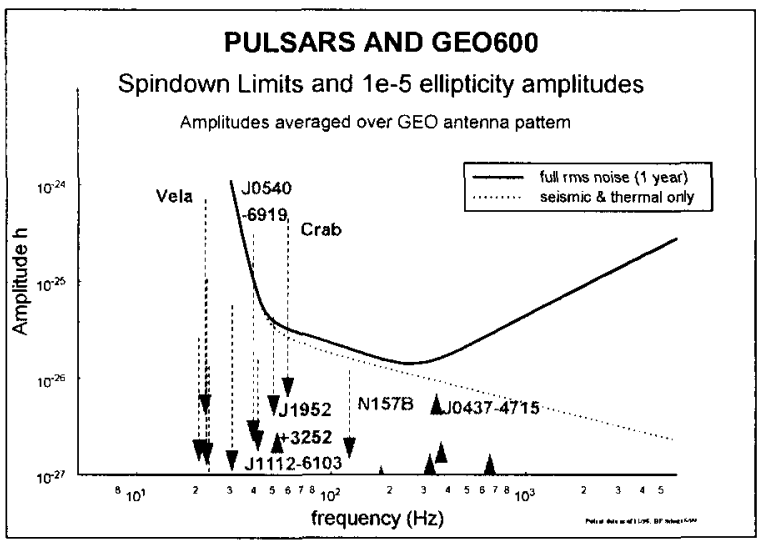

Figure 1. Sensitivity of the GEO600 detector in a 1-year observation compared to the spindown upper limits on the amplitude of radiation from some known pulsars (top of arrow) and to the radiation expected if the ellipticity is $10^{-5}$ (bottom of arrow).

The amplitudes here are much smaller than $10^{-21}$ because the amplitude sensitivity in matched filtering (as in the Fourier transform) improves with the square-root of the number of cycles in the radiation waveform. This sensivitiy gain requires continuous observing for the whole year: gaps reduce sensitivity. Continuous observing is one of the design goals of the interferometers, and even prototypes have attained duty cycles better than 90The broad quadrupolar antenna pattern of the detectors allows one to survey the entire sky with just one observing run.

\section{Specific Gravitational Wave Emission Mechanisms}

A number of radiation mechanisms may operate, alone or in combination.

Crust irregularities would radiate as they spin with the star. The jelly-like crust can sustain some strain, but estimates of its strength vary considerably. It seems that the crust could sustain an ellipticity of at least $10^{-5}$ for long periods of time, as used in Figure 1. The radiation emerges at twice the spin frequency of the star, because the quadrupolar asymmetry that is the effective source is periodic with just half the spin period of the star. Glitches may either relieve excess strain (radiation before the glitch) or induce it (radiation after the glitch). If neutron stars have a solid core, it would probably be much stronger than the 
crust, and a sustainable ellipticity of at least $10^{-3}$ is plausible (the upper limit for the Crab).

An interesting recent theoretical development has been the discovery by $N$. Andersson of the $r$-mode instability of neutron stars (see Owen et al 1998 and references therein). It appers that the instability affects hot neutron stars $\left(T>10^{8} \mathrm{~K}\right)$ that spin faster than about $100-150 \mathrm{~Hz}$, and that it will cause them to spin down to this frequency in a period of about one year. Newly-formed pulsars are much hotter than this, so there should be no young pulsars spinning faster than about $150 \mathrm{~Hz}$. Recycled pulsars are not constrained, because even during accretion they do not get hotter than about $10^{8} \mathrm{~K}$.

If the $r$-mode instability occurs in young neutron stars, then many supernova explosions should be followed by a period of nearly monochromatic radiation lasting of order a year (Owen et al. 1999). The radiation will emerge with a frequency $4 / 3$ times the spin frequency of the star, because that is the frequency of the dominant unstable mode. The amplitude of the radiation is not quite high enough for first-generation detectors to see if the star is in the Virgo Cluster, but a nearer supernova might be seen if we can solve the difficult problem of adequately predicting the signal waveform, ie the spin evolution of the star for the observation period.

Another interesting recent suggestion is that an accreting neutron star could develop a mass asymmetry as a result of the accretion (Bildsten 1998). The idea is that accretion itself may be asymmetric because of the magnetic field. The accreted mass itself spreads out quickly and leaves little asymmetry, but there remains an asymmetry in the star's temperature distribution. This in turn affects the equilibrium composition of the crust: the mean neutron number of the nuclei of the crust depends on the equilibrium temperature. This composition gradient in turn leads to a density gradient, and that emits gravitational radiation at twice the star's spin frequency.

Bildsten proposed this model to explain why observed neutron stars in lowmass X-ray binaries all seem to have the same spin frequency, around $300 \mathrm{~Hz}$ (van der Klis 1998). He showed that the mechanism could radiate enough angular momentum to balance the accreted angular momentum when such a star spins at $300 \mathrm{~Hz}$. Since the gravitational-wave energy radiated is proportional to the energy radiated in X-rays, the observed amplitude can be deduced directly from the observed X-ray flux, independently of the (sometimes uncertain) distance to the source.

The strongest source, on the basis of this model, would be Sco X-1, but it will be difficult to observe with the first instruments, even using the advanced GEO600 technique of narrow-banding.

\section{Detecting Neutron Stars from Gravitational Waves}

There are special difficulties of data analysis when looking for neutron star signals, and they are related to some radio pulsar data analysis problems. The basic data stream is a time series filtered to acoustic frequencies, perhaps to $1.5 \mathrm{kHz}$, and sampled at twice that rate. The signal is too weak to see in the broad-band time-series output. One needs to accumulate signal in one frequency band for at least $10^{7} \mathrm{~s}$ in order to have a reasonable chance to see a signal. 
This contrasts with the $10^{3} \mathrm{~s}$ of a typical radio observation. During this long timescale, phase modulation due to the motion of the Earth and pulsar spindown (if $d P / d t>10^{-14}$ ) must be modelled accurately.

Gravitational waves suffer no dispersion (or absorption), but there are other parameters in the signal model: the angular position, time derivatives of the spin., and any possible binary orbital parameters. They are extracted from the particular phase modulation patterns they produce. The signal model must be accurate to within one cycle of the phase of the wave during the entire observation; if it goes out of phase then it will introduce zero or negative correlation over some of the time-series, and this will reduce sensitivity. If the family of possible correlation templates is large, as it is for an all-sky survey, the required computational power is large, and the significance of any response has to be considered carefully the more trial correlations one looks at, the greater is the chance that pure noise will produce a large correlation (Schutz 1991, 1989). The specific problem of pulsar detection has been discussed extensively in the literature (Brady et al. 1998, Jaranowski et al. 1998)

The gravitational wave equivalent of "pointing" is done in data analysis by removing phase modulation for a given position, correcting for spindown, and then doing a Fourier search. The search for a pulsar of period $P$ can be confined to periods near $P, 3 P / 4$, and $P / 2$. Getting good data from radio $/ \mathrm{X} / \gamma /$ optical observations is critical: positions, $P$, and $d P / d t$, and glitches are all needed to build good templates for matched filtering.

For a pointed observation with good parameter data the computer work is trivial. However, if the target object is a binary and the orbital parameters are poorly known, then then the efficient but sub-optimal orbital modulation search method of Ransom (private communication 1999), developed for radio searches, could also be applied to gravitational wave data.

Suitable objects for pointed observations where some or all of the parameters are known include known pulsars, X-ray binaries and novae, unidentified X-ray point sources, and suspect Thorne-Zytkov objects. Any of these could harbour a neutron star that is radiating continuously.

Large-area surveys can be done by data analysis of a single observation run, but they are computationally expensive. Target areas would include the Galactic plane, globular clusters, and supernova remnants. Unfortunately, a a wide-area survey down to the $1 \sigma$ noise level attainable in a 4 -month observation requires examining $10^{13}$ or more parameter sets, which would require a teraflopclass computer working full time on the data analysis (Schutz 1991, Brady et al. 1998). But with so many searches, there is no significance to a response at the few- $\sigma$ level. A suitable signficance threshold is $10 \sigma$ on the amplitude, which can be reached with hierarchical algorithms that will run on a cluster of workstations with a capacity of 20 Gflops (Schutz 1998, Papa \& Schutz 1999, Brady $\&$ Creighton 1998). These algorithms might be interesting to radio astronomers doing pulsar searches with large parameter spaces.

\section{Conclusion - Gravitational Wave Astronomy}

As first-generation gravitational wave detectors near completion, radio astronomers and gravitational wave astronomers have much to talk to one another 
about. Gravitational wave observations can supply key new information, such as the spin axis orientation, or the discovery of hidden or distant neutron stars. They also require the best possible input information from radio, optical, and $\mathrm{X}$-ray observations. And the new computer methods being developed for gravitational wave searches may also offer improvements in sensitivity for radio searches for binaries and other large-parameter-set systems.

\section{References}

Bildsten, L. 1998, ApJ, 501, L89

Blanchet, L., Damour, T., Iyer, B. R., et al. 1995, Phys Rev Lett, 74, 3515

Brady, P. R., and Creighton, T., 1998, preprint, gr-qc/9812014.

Brady, P.R., Creighton, T., Cutler, C. and Schutz, B.F. 1998, Phys Rev, 57, 2101

Danzmann, K. 1995 in Coccia, E., Pizzella, G., Ronga, F., eds., Gravitational Wave Experiments (World Scientific Publishing Co. Pte. Ltd., Singapore) 100

Giazotto, A., 1995 in Coccia, E., Pizzella, G., Ronga, F., eds., Gravitational Wave Experiments (World Scientific Publishing Co. Pte. Ltd., Singapore) 86

Helström, C.W. 1968 Statistical Theory of Signal Detection, 2nd ed. (Pergamon Press, London).

Jaranowski, P., Krolak, A., Schutz, B.F., 1998, Phys Rev, D5806, 3001

Nicholson, D., Dickson, C.A., Watkins, W.J., et al. 1996, Physics Letters A, 218, 175

Owen, B., Lindblom, L., Cutler, C., Schutz, B.F., Vecchio, A., Andersson, N. 1999, Phys Rev, D58, 084020

Papa, M.-A., \& Schutz, B. F., in Gravitational Waves and Experimental Gravity (Proceedings of Moriond 1999) (Editions Frontieres, Orsay, 1999); grqc/9905018.

Raab, F. J. 1995 in Coccia, E., Pizzella, G., Ronga, F., eds., Gravitational Wave Experiments (World Scientific Publishing Co. Pte. Ltd., Singapore) 80.

Schutz, B.F., ed., 1989 Gravitational Wave Data Analysis (Kluwer, Dordrecht).

Schutz, B.F., 1991 "Data Processing Analysis and Storage for Interferometric Antennas" in The Detection of Gravitational Waves ed. D.G. Blair (Cambridge University Press, Cambridge) 406-452

Schutz, B. F., in Davier, M., and Hello, P., Proceedings of Second Gravitational Wave Data Analysis Workshop (Editions Frontiers, Orsay, 1998), 113

Strain, K.A., \& Meers, B.J. 1991, Phys Rev, 66, 1391

Taylor, J. H. 1994, Rev. Mod. Phys., 66, 711

Tsubono, K., 1995, in Coccia, E., Pizzella, G., Ronga, F., eds., Gravitational Wave Experiments (World Scientific Publishing Co. Pte. Ltd., Singapore) 114.

van der Klis, M. 1998, Phys Scripta,T77, 69 